J. Campos · E.N. Dancer · R. Ortega

\title{
Dynamics in the neighbourhood of a continuum of fixed points ${ }^{\star}$
}

Received: October 30, 2000; in final form: February 20, 2001

Published online: December 19, 2001 - (C) Springer-Verlag 2001

\section{Introduction}

Let $\mathbb{D}$ be the closed unit disk of $\mathbb{R}^{2}$ and let $h$ be a homeomorphism from $\mathbb{D}$ onto $\mathbb{D}$ which is orientation-preserving and such that all the fixed points are on the boundary, that is

$$
\operatorname{Fix}(h) \subset \partial \mathbb{D}=\mathbb{S}^{1}
$$

Under these conditions it was proved in [4] that $h$ has trivial dynamics. This means that the $\omega$-limit set of every orbit $\left\{h^{n}(p)\right\}$ is a continuum included in Fix $(h)$. Some examples were constructed in [4] where $h$ was a $C^{\infty}$-diffeomorphism and the $\omega$ limit set was an arc or even the whole circle $\mathbb{S}^{1}$. The next result will show that this cannot happen if $h$ is real analytic. We notice that all this is reminiscent of the theory of limit sets for gradient-like vector fields (see $[9,6]$ ).

Theorem 1. Assume that $h$ is an orientation-preserving $C^{\omega}$-diffeomorphism and

$$
\operatorname{Fix}(h) \subset \partial \mathbb{D} \text {. }
$$

Then every orbit converges to a fixed point of $h$.

In the assumptions of this theorem it is clear that $F i x(h)$ is either finite on coincides with $\mathbb{S}^{1}$. In the first case the proof follows directly from [4] and so the proof of Theorem 1 is reduced to the case $F i x(h)=\mathbb{S}^{1}$.

Another situation where one is lead to the study of two-dimensional diffeomorphisms having a continuum of fixed points appears in the study of degenerate

J. Campos, R. Ortega: Departamento de Matemática Aplicada, Facultad de Ciencias, Universidad de Granada, 18071-Granada, Spain

e-mail: jcampos@ugr.es, rortega@ugr.es

E.N. Dancer: School of Mathematics and Statistics, University of Sydney, Sydney, NSW 2006, Australia, e-mail: normd@maths . usyd. edu . au

* E.N. Dancer is partially supported by the Australian Research Council. J. Campos and R. Ortega by DGES PB98-1294 (Spain). 
equations of pendulum type (see [8]). Consider the differential equation

$$
\ddot{x}+V_{x}(t, x)=0,
$$

where the potential $V: \mathbb{R} \times \mathbb{R} \rightarrow \mathbb{R}$ is periodic in both variables, say with common period $2 \pi$. This equation will be called degenerate if the perturbed equation

$$
\ddot{x}+V_{x}(t, x)=s
$$

has no $2 \pi$-periodic solutions for any value of the parameter $s \in \mathbb{R}, s \neq 0$. It can be proved that a degenerate equation always has a continuum of $2 \pi$-periodic solutions and so the corresponding Poincaré map is a diffeomorphism of the plane having a continuum of fixed points. There have been several papers on heteroclinic solutions of (1), see [10,3]. The next result shows that degeneracy is an obstruction for the existence of such solutions.

Theorem 2. Assume that $V \in C^{3}$ and (1) is degenerate. Then every solution which is bounded in the future is a $2 \pi$-periodic solution.

Under milder conditions of regularity on $V$, it was proved in Theorem 3.2 of [8] that almost all solutions are unbounded in the future when (1) is degenerate. For smooth potentials the previous result improves that theorem because it is known that for a degenerate equation the set of periodic solutions has measure zero.

In this paper we discuss some dynamical properties of diffeomorphisms in the plane having a continuum of fixed points. In particular we analyse the dynamical information carried by the linearization at the continuum. This study will lead us to prove Theorems 1 and 2. As we shall see, both proofs are based on similar ideas.

Campos and Ortega thank Enrico Serra and Massimo Tarallo for conversations on the pendulum equation where they posed the question that lead to Theorem 2. Dancer thanks Campos and Ortega for their hospitality during his visit to Granada in January 2000.

\section{Linearization at a continuum of fixed points}

Let $f: \mathbb{R}^{2} \rightarrow \mathbb{R}^{2}$ be an orientation-preserving diffeomorphism of class $C^{1}$ and assume that there is a segment of fixed points, say

$$
\Gamma \subset \operatorname{Fix}(f), \quad \Gamma=I \times\{0\},
$$

where $I$ is an open interval in $\mathbb{R}$.

The derivative of $f$ at $\Gamma$ takes the form

$$
f^{\prime}(x, 0)=\left(\begin{array}{ll}
1 & \beta(x) \\
0 & \alpha(x)
\end{array}\right), \quad x \in I,
$$

where $\alpha$ and $\beta$ are continuous functions on $I$ and $\alpha$ is positive everywhere. The local dynamics around a point of $\Gamma$ where $\alpha \neq 1$ can be understood using the results of [7]. We state a result which can be extracted from [7]. 
Proposition 3. Assume that $\alpha(\tilde{x}) \neq 1$ for some $\tilde{x} \in I$. Then there exists a neighbourhood $U \subset \mathbb{R}^{2}$ of $(\tilde{x}, 0)$ and a local homeomorphism $G: U \rightarrow \mathbb{R}^{2}$ such that

$$
G(x, 0)=(x, 0)
$$

and

$$
G^{-1} \circ f \circ G(x, y)=(x, \tilde{\alpha} y) .
$$

These two identities hold in some small neighbourhood of $(\tilde{x}, 0)$ and $\tilde{\alpha}$ is just $\alpha(\tilde{x})$. We split $\Gamma$ in the form

$$
\Gamma_{s}=\{(x, 0): \alpha(x)<1\}, \quad \Gamma_{u}=\{(x, 0): \alpha(x)>1\}, \quad \Gamma_{0}=\Gamma-\left(\Gamma_{s} \cup \Gamma_{u}\right) .
$$

The previous proposition implies that the points in $\Gamma_{s}$ are Lyapunov stable and attract some orbits while the points in $\Gamma_{u}$ are unstable and do not attract any orbit.

We shall say that a point $\left(x_{0}, y_{0}\right) \in \mathbb{R}^{2}-\Gamma$ is attracted by $\Gamma$ if the positive semi-orbit $\left\{f^{n}\left(x_{0}, y_{0}\right)\right\}_{n \geq 0}$ is bounded and the $\omega$-limit set $L_{\omega}\left(x_{0}, y_{0}\right)$ is contained in $\Gamma$. Of course $\Gamma$ attracts orbits as soon as $\Gamma_{s}$ is non-empty. The next result gives some insight on how orbits can approach $\Gamma$.

Lemma 4. Let $\left\{\left(x_{n}, y_{n}\right)\right\}_{n \in \mathbb{Z}}$ denote the orbit starting at $\left(x_{0}, y_{0}\right) \in \mathbb{R}^{2}-\Gamma$ and assume that it is attracted by $\Gamma$. Then $y_{n}$ has a definite sign for large $n$. More precisely, there exists $v>0$ such that either $y_{n}>0$ for all $n \geq v$ or $y_{n}<0$ for all $n \geq v$.

In addition assume now that there is some $\tilde{x} \in I$ such that $(\tilde{x}, 0) \in L_{\omega}\left(x_{0}, y_{0}\right)$ and $\beta(\tilde{x}) \neq 0$. Then $x_{n}$ is eventually monotone. This means that, for some $v_{1}>0$, either $x_{n}>x_{n+1}$ for all $n \geq v_{1}$ or $x_{n}<x_{n+1}$ for all $n \geq v_{1}$.

Proof. Since $L_{\omega}\left(x_{0}, y_{0}\right)$ is a compact subset of $\Gamma$, the definition of attraction implies that $y_{n} \rightarrow 0$ as $n \rightarrow+\infty$. We are going to prove that $y_{n}$ has a definite sign when $n$ is large. Otherwise we could find an arbitrary large $n$ such that $y_{n}$ and $y_{n+1}$ have opposite signs, say $y_{n}>0$ and $y_{n+1}<0$. Notice that $y_{n}$ cannot vanish for large $n$. Then we could construct a very small arc $\gamma$ passing through $\left(x_{n}, y_{n}\right)$ and with two different extreme points $\left(e_{i}, 0\right)$ and $\left(e_{f}, 0\right)$ belonging to $\Gamma$ and such that

$$
\dot{\gamma} \subset\{y>0\}, \quad f(\dot{\gamma}) \subset\{y<0\} .
$$

Let $\lambda$ be the loop composed by arc $\gamma$ and the segment in $\Gamma$ joining $\left(e_{i}, 0\right)$ and $\left(e_{f}, 0\right)$. For instance, $\lambda$ could be taken as a small triangle. If we give an orientation to the loop, then it is intuitively clear that the transformed loop $f(\lambda)$ will have the reversed orientation. This is not possible because $f$ is orientation-preserving.

Now we prove the second part of the lemma. For each $(x, y) \in I \times \mathbb{R}$ the first component of $f=\left(f_{1}, f_{2}\right)$ can be expressed in the form

$$
f_{1}(x, y)=x+y g(x, y),
$$

where $g$ is continuous and $g(x, 0)=\beta(x)$. For large $n$ the sequence $\left(x_{n}, y_{n}\right)$ lies on $I \times \mathbb{R}$ and we denote by $M$ a fixed constant for which

$$
\left|g\left(x_{n}, y_{n}\right)\right| \leq M, \quad n \geq N .
$$


We are also going to assume that $y_{n}$ is positive and $\beta(\tilde{x})>0$ and we shall prove that $x_{n}$ is eventually increasing. The other cases are treated similarly.

Let $\epsilon>0$ be such that

$$
g(x, y)>0 \quad \forall(x, y) \in[\tilde{x}-\epsilon, \tilde{x}+\epsilon] \times[0, \epsilon]
$$

and let $\epsilon^{\star}$ be defined as

$$
\epsilon^{\star}=\min \left\{\frac{\epsilon}{2}, \frac{\epsilon}{2 M}\right\} .
$$

We can find $N_{1}>0$ such that

$$
y_{n} \leq \epsilon^{\star} \text { if } n \geq N_{1}
$$

and

$$
x_{N_{1}} \in[\tilde{x}-\epsilon, \tilde{x}+\epsilon] .
$$

We shall prove that $x_{n}$ is increasing for $n \geq N_{1}$. To do this we verify that $x_{n}$ remains in the interval $[\tilde{x}-\epsilon, \tilde{x}+\epsilon]$ for $n \geq N_{1}$. This is sufficient because (2) and (4) imply that $x_{n+1}>x_{n}$ if $x_{n} \in[\tilde{x}-\epsilon, \tilde{x}+\epsilon]$.

By a contradiction argument let us assume that $x_{n}$ does not remain in $[\tilde{x}-\epsilon$, $\tilde{x}+\epsilon]$. Then it must exit by the right and, since $(\tilde{x}, 0)$ is in $L_{\omega}\left(x_{0}, y_{0}\right)$, we can find $m>N_{1}$ such that $x_{m} \in\left(-\infty, \tilde{x}+\epsilon^{\star}\right)$ but $x_{m-1} \geq \tilde{x}+\epsilon^{\star}$. We distinguish two cases:

(i) $x_{m-1} \leq \tilde{x}+\epsilon$. Again (2) and (4) imply that $x_{m}>x_{m-1}$ and this is impossible.

(ii) $x_{m-1}>\tilde{x}+\epsilon$. We combine (2) with (3) to obtain

$$
x_{m} \geq x_{m-1}-M \epsilon^{\star}>\tilde{x}+\epsilon-M \epsilon^{\star} \geq \tilde{x}+\epsilon^{\star} .
$$

This is not compatible with the definition of $m$.

Next we present a result describing the structure of the limit set.

Proposition 5. Assume that $\left(x_{0}, y_{0}\right) \in \mathbb{R}^{2}-\Gamma$ is attracted by $\Gamma$. Then either $L_{\omega}\left(x_{0}, y_{0}\right)$ is a singleton contained in $\Gamma_{s} \cup \Gamma_{0}$ or it is a continuum lying in $\tilde{\Gamma}_{0}$. Here $\tilde{\Gamma}_{0}$ is the subset of $\Gamma_{0}$ defined as

$$
\tilde{\Gamma}_{0}=\{(x, 0): \alpha(x)=1, \beta(x)=0\} .
$$

In particular $L_{\omega}\left(x_{0}, y_{0}\right)$ is a singleton when $\tilde{\Gamma}_{0}$ is totally disconnected.

Proof. From Proposition 2.5 in [4] we know that $L_{\omega}\left(x_{0}, y_{0}\right)$ is connected. The rest of the proof follows from the previous discussions.

We now discuss the case $\Gamma=\Gamma_{0}$; that is, $\alpha \equiv 1$. This is certainly non-generic but it always occurs when $f$ is area-preserving. When $\alpha \equiv 1$ the regularity of $f$ becomes relevant.

Theorem 6. Assume that $f$ is of class $C^{2}$ and $\alpha(x)=1$ for each $x \in I$. In addition assume that some point $\left(x_{0}, y_{0}\right)$ outside the continuum is attracted by $\Gamma$. Then

$$
L_{\omega}\left(x_{0}, y_{0}\right) \subset\{(x, 0) \in \Gamma: \beta(x)=0\} .
$$


Remarks. 1. The result is not valid if one assumes only $f \in C^{1, \alpha}$ for some $\alpha \in(0,1)$. We shall present an example after the proof of the theorem.

2. The previous result implies that if $\alpha \equiv 1$ and $\beta$ is positive or negative then $\Gamma$ cannot attract any orbit. When $\beta$ vanishes somewhere one needs to consider the higher-order terms of $f$ to decide whether $\Gamma$ attracts orbits or not. As an example consider a family of diffeomorphisms $\left\{f_{\lambda}\right\}_{\lambda \in \mathbb{R}}$ satisfying

$$
f_{\lambda}:\left\{\begin{array}{l}
x_{1}=x-x y \\
y_{1}=y+\lambda y^{3}
\end{array}\right.
$$

in some small neighbourhood of the origin $|x|,|y| \leq \epsilon$. In this case we have the continuum $\Gamma=(-\epsilon, \epsilon) \times\{0\}$ and $\alpha \equiv 1, \beta(x)=-x$. We notice that this continuum attracts orbits if $\lambda<0$ but not if $\lambda \geq 0$. Actually, if $\Gamma$ attracts an orbit $\left(x_{n}, y_{n}\right)$ one has $y_{n} \rightarrow 0$ and $0<\left|y_{n}\right|<\epsilon, y_{n+1}=y_{n}+\lambda y_{n}^{3}$ for large $n$. This is not possible if $\lambda \geq 0$. For $\lambda<0$ the points $\left(x_{0}, y_{0}\right)$ with $\left|x_{0}\right|<\epsilon$ and $0<y_{0}<\min \left\{\epsilon, 1,|\lambda|^{-1 / 2}\right\}$ are attracted by $\Gamma$. To check this notice that $y_{n}$ is a decreasing sequence converging to 0 and $x_{n}=x_{0} \prod_{k=0}^{n-1}\left(1-y_{k}\right)$.

Proof. When $L_{\omega}\left(x_{0}, y_{0}\right)$ is a continuum the result is a consequence of Proposition 5. From now on we shall assume that the sequence $\left(x_{n}, y_{n}\right)$ converges to some point in $\Gamma$. Also, in view of Lemma 4 , we can assume that $y_{n}$ has a definite sign, say

$$
y_{n}>0 \quad \text { if } n \geq 0 \text {. }
$$

We first claim that

$$
\sum_{n \geq 0} y_{n}=+\infty
$$

To prove this we use that $f$ is $C^{2}$ and denote by $C>0$ a bound of $\left|\frac{\partial^{2} f_{2}}{\partial y^{2}}\right|$ on some convex region containing the semi-orbit $\left\{\left(x_{n}, y_{n}\right)\right\}_{n \geq 0}$. From Taylor's expansion

$$
f_{2}(x, y)-f_{2}(x, 0)-\frac{\partial f_{2}}{\partial y}(x, 0) y=\frac{1}{2} \frac{\partial^{2} f_{2}}{\partial y^{2}}(x, \xi) y^{2}
$$

we deduce that

$$
\left|y_{n+1}-y_{n}\right| \leq \frac{C}{2} y_{n}^{2}, \quad n \geq 0 .
$$

We shall prove that any solution of this difference inequality satisfying $y_{n} \rightarrow 0$ and (5) cannot go to zero faster than $\frac{1}{n}$. The claim (6) follows from this.

To do this we use a comparison argument. Consider the sequence $z_{n}=\frac{b}{n}$ where $b>0$ will be determined later. It satisfies

$$
z_{n+1} \leq z_{n}-\frac{1}{2 b} z_{n}^{2}
$$

For $b \leq \frac{1}{C}$ the sequence $\left\{z_{n}\right\}$ is a lower solution of the difference equation

$$
r_{n+1}=f\left(r_{n}\right), \quad f(r)=r-\frac{C}{2} r^{2} .
$$


From (7) we deduce that $\left\{y_{n}\right\}$ is an upper solution. Since $f$ is increasing on $\left[0, C^{-1}\right]$, comparison methods will work for solutions lying on that interval. We select $N$ large enough so that $y_{n} \leq C^{-1}$ if $n \geq N$. By restricting the size of $b$ we can also assume that $y_{N} \geq z_{N}$ and so $y_{n} \geq z_{n}$ for large $n$.

After proving (6) we are ready to complete the proof of the theorem. As before we apply Taylor's formula (now for $f_{1}$ ) and obtain

$$
\left|x_{n+1}-x_{n}-\beta\left(x_{n}\right) y_{n}\right| \leq C_{1} y_{n}^{2},
$$

where $C_{1}$ is a fixed constant. By a contradiction argument assume that $\beta$ is not zero at the limit of $\left(x_{n}, y_{n}\right)$. We shall assume that it is positive (the case of $\beta$ being negative is similar). Then we find $\beta_{\star}>0$ such that, for large $n$,

$$
\beta\left(x_{n}\right) \geq \beta_{\star}
$$

and so,

$$
x_{n+1} \geq x_{n}+\beta\left(x_{n}\right) y_{n}-C_{1} y_{n}^{2} \geq x_{n}+\frac{\beta_{\star}}{2} y_{n} .
$$

Thus we can apply (6) to deduce that $x_{n} \rightarrow+\infty$. This is not consistent with the assumptions.

Example. Given $\alpha \in(0,1)$ consider a function $g \in C^{1, \alpha}(\mathbb{R})$ satisfying $g^{\prime}(y)>0$ $\forall y \in \mathbb{R}, g(-\infty)=-\infty, g(+\infty)=+\infty$ and

$$
g(y)=\frac{y}{\left(1+|y|^{\alpha}\right)^{1 / \alpha}} \quad \text { if }|y| \leq 1 .
$$

For large $c>0$ the sequence $y_{n}=\frac{1}{(n+c)^{1 / \alpha}}$ is a solution of

$$
y_{n+1}=g\left(y_{n}\right) \text {. }
$$

The diffeomorphism

$$
f(x, y)=(x+y, g(y))
$$

is of class $C^{1, \alpha}$ and contains the continuum of fixed points $\Gamma=\mathbb{R} \times\{0\}$. Since $g^{\prime}(0)=1$ one has $\alpha \equiv 1$. Except for the regularity, $f$ satisfies the conditions of Theorem 6 . However $\beta \equiv 1$ does not vanish and $\Gamma$ attracts orbits of the type

$$
x_{n}=x_{0}+\sum_{k=0}^{n-1} \frac{1}{(k+c)^{1 / \alpha}}, \quad y_{n}=\frac{1}{(n+c)^{1 / \alpha}},
$$

for small $x_{0}$ and large $c$.

We are now ready to prove one of the results stated in the introduction.

Proof of Theorem 2. The degeneracy of the equation leads to the existence of a continuum of periodic solutions which attract any possible bounded solution. More precisely, there exists a function $\Phi=\Phi(t, \xi), \Phi: \mathbb{R} \times \mathbb{R} \rightarrow \mathbb{R}$, with the properties listed below:

(i) $\Phi(t+2 \pi, \xi)=\Phi(t, \xi), \quad \Phi(t, \xi+2 \pi)=\Phi(t, \xi)+2 \pi, \quad \Phi(0, \xi)=\xi$;

(ii) for each $\xi \in \mathbb{R}, \Phi(\cdot, \xi)$ is a periodic solution of (1); 
(iii) $\Phi$ and $\frac{\partial \Phi}{\partial t}$ are of class $C^{2}$;

(iv) given a solution $x(t)$ of (1) which is bounded in $[0, \infty)$, there exists $\xi \in \mathbb{R}$ such that

$$
|x(t)-\Phi(t, \xi)|+\left|\dot{x}(t)-\frac{\partial \Phi}{\partial t}(t, \xi)\right| \rightarrow 0 \quad \text { as } t \rightarrow+\infty .
$$

All these properties follow directly from the results in [8] excepting (iii). This is a consequence of the proof of Lemma 4.1 in the same paper. In particular we recall that the function

$$
\varphi(\xi):=\frac{\partial \Phi}{\partial t}(0, \xi)
$$

is in $C^{2}(\mathbb{R})$.

Given $\xi, \eta \in \mathbb{R}$, let $z(t ; \xi, \eta)$ denote the solution of (1) with $z(0)=\xi, \dot{z}(0)=\eta$. The Poincaré map

$$
P:(\xi, \eta) \mapsto\left(\xi_{1}, \eta_{1}\right), \quad \xi_{1}=z(2 \pi ; \xi, \eta), \eta_{1}=\dot{z}(2 \pi ; \xi, \eta)
$$

is a $C^{2}$-diffeomorphism of $\mathbb{R}^{2}$ and the curve $\eta=\varphi(\xi)$ is composed of fixed points. Next we perform a change of variables mapping this curve onto the horizontal axis, namely

$$
\Psi:(\xi, \eta) \mapsto(x, y), \quad x=\xi, \quad y=\eta-\varphi(\xi) .
$$

In the new variables our diffeomorphism becomes $f=\Psi \circ P \circ \Psi^{-1}$. It is orientationpreserving and contains the continuum of fixed points $\Gamma=\mathbb{R} \times\{0\}$. Next we discuss the linearization of $f$ at $\Gamma$. Since $\Psi$ and $P$ are area-preserving, our map $f$ has also this property and so $\alpha \equiv 1$. The coefficient $\beta(x)$ satisfies

$$
\beta(x)=\frac{\partial f_{1}}{\partial y}(x, 0)=\frac{\partial z}{\partial \eta}(2 \pi ; x, \varphi(x))
$$

and the formula (4.7) in the proof of Lemma 4.1 in [8] implies that $\beta$ is positive everywhere. We can now apply Theorem 6 to $f$ to deduce that $\Gamma$ does not attract any point $(x, y)$ with $y \neq 0$. Property (iv) stated above now shows that the only solutions bounded in the future are those of the family $\Phi$.

Remark. The previous proof goes through if one assumes only that the potential $V$ is continuous and has continuous partial derivatives $V_{x}, V_{x x}$ and $V_{x x x}$.

\section{Free homeomorphisms and the proof of Theorem 1}

To prove Theorem 1 we shall combine some of the ideas of the previous section with the theory of free homeomorphisms in two dimensions. We review some facts of this theory and refer to [1], [2] and [5] for more information.

For our purposes it will be sufficient to work on the space

$$
\mathbb{H}=\left\{(x, y) \in \mathbb{R}^{2}: y \geq 0\right\} .
$$


This is a manifold with boundary and we shall employ the usual notations $\operatorname{int}(\mathbb{H})$ and $\partial \mathbb{H}$ for the interior and the boundary.

Given an orientation-preserving homeomorphism $g$ of $\mathbb{H}$, we shall say that $g$ is free if, given an arbitrary topological disk $D \subset \operatorname{int}(\mathbb{H})$ with $g(D) \cap D=\emptyset$, then $g^{n}(D) \cap D=\emptyset$ for each $n \geq 2$.

It was proved in Lemma 3.1 in [2] that this property of successive empty intersection is inherited by an arbitrary continuum. More precisely, if $g$ if free and $K \subset \operatorname{int}(\mathbb{H})$ is compact, connected and satisfies $g(K) \cap K=\emptyset$ then $g^{n}(K) \cap K=\varnothing$ for each $n \geq 2$.

In general it can be difficult to determine if a homeomorphism is free, but if $g$ has no fixed points in $\operatorname{int}(\mathbb{H})$ then it is automatically free. This is a consequence of Brouwer's Lemma [1] and [2] because $\operatorname{int}(\mathbb{H})$ is homeomorphic to $\mathbb{R}^{2}$.

The next result presents an obstruction to freeness.

Lemma 7. Let $g$ be an orientation-preserving diffeomorphism of class $C^{\omega}$. Assume that

$$
\partial \mathbb{H} \subset \operatorname{Fix}(g),
$$

and there exists $\left(x_{0}, y_{0}\right) \in \operatorname{int}(\mathbb{H})$ such that

$$
[0,1] \times\{0\} \subset L_{\omega}\left(x_{0}, y_{0}\right)
$$

Then $g$ is not free.

Remark. It must be observed that in this lemma we are not assuming that the semi-orbit $\left\{\left(x_{n}, y_{n}\right)\right\}_{n \geq 0}$ is bounded. In particular $L_{\omega}\left(x_{0}, y_{0}\right)$ could be unbounded.

Proof. It will be sufficient to prove the existence of a continuum $K \subset \operatorname{int}(\mathbb{H})$ such that $g(K) \cap K=\emptyset$ but $g^{d}(K) \cap K \neq \emptyset$ for some $d>1$. We assume for the moment that the following holds:

Claim. There exists an interval $[a, b] \subset[0,1]$, a number $\epsilon>0$ and integers $q>p+1, p \geq 1$, such that

i) $\left(x_{p}, y_{p}\right),\left(x_{q}, y_{q}\right) \in[a, b] \times(0, \epsilon]$;

ii) $x_{p}<x_{q}<x_{p+1}$;

iii) $\frac{\partial g_{1}}{\partial x}(x, y)>0$ and $g_{1}(x, y)>x$ if $(x, y) \in[a, b] \times(0, \epsilon]$.

With the claim it is easy to construct $K$. Let $K_{1}$ be the segment joining $\left(x_{p}, y_{p}\right)$ with $\left(x_{q}, y_{p}\right)$ and let $K_{2}$ be the segment joining $\left(x_{q}, y_{p}\right)$ to $\left(x_{q}, y_{q}\right)$. Define $K=K_{1} \cup K_{2}$. The point $\left(x_{q}, y_{q}\right)$ belongs to $g^{q-p}(K) \cap K$. Let us now prove that $K$ and $g(K)$ do not intersect. Given $(x, y) \in K_{1}$ we know that $x \geq x_{p}$ and $y=y_{p}$. Thus

$$
g_{1}\left(x, y_{p}\right) \geq g_{1}\left(x_{p}, y_{p}\right)=x_{p+1}>x_{q}
$$

and $g(x, y)$ cannot lie on $K$. In a similar way, given $(x, y) \in K_{2}$ we know that $x=x_{q}$ and so

$$
g_{1}\left(x_{q}, y\right)>x_{q}
$$


It remains to prove that the claim holds. To do this we first notice that $g_{1}$ must depend upon $y$, for otherwise $g_{1}$ would be a function of $x$ and $L_{\omega}\left(x_{0}, y_{0}\right)$ should be contained in $\left\{x_{0}\right\} \times \mathbb{R}$. This is not consistent with the assumptions. Let us now assume that $g_{1}$ depends on $y$ and let $m \geq 1$ be the first positive integer for which

$$
\beta_{m}(x):=\frac{\partial^{m} g_{1}}{\partial y^{m}}(x, 0)
$$

is not identically zero. By expanding in $y$,

$$
g_{1}(x, y)=x+\beta_{m}(x) y^{m}+\Phi(x, y) y^{m+1} .
$$

Let us now choose an interval $[a, b] \subset[0,1]$ where $\beta_{m}$ is positive (the case $\beta_{m}$ negative is similar). We consider fixed constants

$$
\begin{gathered}
\delta=\min \left\{\beta_{m}(x): x \in[a, b]\right\}, \quad \Delta=\max \left\{\left|\beta_{m}^{\prime}(x)\right|: x \in[a, b]\right\}, \\
M=\max \{|\Phi(x, y)|: \quad(x, y) \in[a, b] \times[0,2]\}, \\
N=\max \left\{\left|\frac{\partial \Phi}{\partial x}(x, y)\right|: \quad(x, y) \in[a, b] \times[0,2]\right\},
\end{gathered}
$$

and we select $\epsilon>0$ satisfying

$$
\begin{gathered}
\delta>M \epsilon, \\
1>\epsilon^{m} \Delta+\epsilon^{m+1} N .
\end{gathered}
$$

We choose a point of the orbit $\left(x_{p}, y_{p}\right)$ lying in the open set $(a, b) \times(0, \epsilon)$. From the assumptions it is clear that there are infinitely many such points. From (8) and (9),

$$
x_{p+1}>x_{p}+(\delta-M \epsilon) y_{p}^{m}>x_{p} .
$$

Define $a^{\star}=x_{p}$ and $b^{\star}=\min \left\{b, x_{p+1}\right\}$. Then $a<a^{\star}<b^{\star} \leq b$ and the set $\left(a^{\star}, b^{\star}\right) \times\{0\}$ is inside $L_{\omega}\left(x_{0}, y_{0}\right)$. This allows us to find another point in the orbit $\left(x_{q}, y_{q}\right)$ with $q>p$ and such that the conclusions i) and ii) of the claim hold. Now iii) can be deduced from (8) and (9), (10).

Proof of Theorem 1. The results of [4] imply that the $\omega$-limit set of any orbit must be a connected subset of Fix $(h)$. Let us argue by contradiction and assume that there is an orbit $\left\{h^{n}(p)\right\}_{n \in \mathbb{Z}}, p \in \operatorname{int}(\mathbb{D})$, whose limit set contains an arc $\gamma$ of $\mathbb{S}^{1}$. Then $\gamma \subset \operatorname{Fix}(h)$ and the identity principle implies that Fix $(h)=\mathbb{S}^{1}$. Next we take a real analytic change of variables $\psi$ from $\mathbb{D}$ onto $\mathbb{H} \cup\{\infty\}$ and such that the the arc $\gamma$ is mapped onto $[0,1] \times\{0\}$. For instance we could compose a Mobius transformation with an $\mathbb{R}$-linear map. We intend to apply Lemma 7 to $g=\psi \circ h \circ \psi^{-1}$. First we observe that some point of $\mathbb{S}^{1}$ has to go to $\infty$ and so the orbit $\left\{g^{n}(\psi(p))\right\}$ could be unbounded. This is not an obstacle for the application of Lemma 7. By construction, the limit set of this orbit contains the continuum $[0,1] \times\{0\}$. Since $g(\infty)=\infty$ we can restrict $g$ to $\mathbb{H}$ and it becomes an orientationpreserving analytic diffeomorphism to which Lemma 7 applies. Thus $g$ is not free and, as we noticed before, Brouwer's Lemma implies that it has a fixed point in $\operatorname{int}(\mathbb{H})$. The corresponding fixed point of $h$ lies in $\operatorname{int}(\mathbb{D})$ and this is in contradiction with the assumptions of the theorem. 
Remark. Consider the periodic system of three competing species:

$$
\dot{u}_{i}=u_{i} F_{i}\left(t, u_{1}, u_{2}, u_{3}\right), \quad i=1,2,3 .
$$

We refer to [4] for the precise assumptions. We shall say that there is extinction for this system if, for any positive solution $\left(u_{1}(t), u_{2}(t), u_{3}(t)\right)$, at least one of the species $u_{i}(t)$ tends to 0 as $t \rightarrow+\infty$. An obvious necessary condition for extinction is the non-existence of positive periodic solutions. In general this condition is not sufficient, but it seems plausible that it could characterize extinction within the class of analytic systems (including the classical Lotka-Volterra model).

It is well known that the dynamics of a competitive system can be reduced to the study of a certain homeomorphism $h$ defined on a topological disk $D$. In this context the non-existence of positive periodic solutions is equivalent to

$$
F i x(h) \subset \partial D,
$$

and the extinction is implied by the convergence of all orbits to points in $\partial D$. These facts were exploited in [4] to obtain sufficient conditions for extinction. Now it is tempting to try to employ Theorem 1 to prove the previously mentioned conjecture about extinction. This is unclear because we cannot guarantee that $D$ and $h$ are analytic even if the functions $F_{i}$ are polynomials with respect to $u$.

In Theorem 1, we can allow the analyticity to fail at isolated points on the boundary and we do not need the analyticity in the interior.

\section{References}

1. Brown, M.: A new proof of Brouwer's lemma on translation arcs. Houston J. Math. 10, 35-41 (1984)

2. Brown, M.: Homeomorphisms of two-dimensional manifolds. Houston J. Math. 11, 455-469 (1985)

3. Calanchi, M., Serra, E.: Homoclinic solutions to periodic motions in a class of reversible equations. Calc. Var. PDEs 9, 157-184 (1999)

4. Campos, J., Ortega, R., Tineo, A.: Homeomorphisms of the disk with trivial dynamics and extinction of competitive systems. J. Diff. Equs. 138, 157-170 (1997)

5. Guillou, L.: Théorème de translation plane de Brouwer et généralisations du théorème de Poincaré-Birkhoff. Topology 33, 331-351 (1994)

6. Haraux, A., Jendoubi, M.A.: Convergence of solutions of second-order gradient-like systems with analytic nonlinearities. J. Diff. Equs. 144, 313-320 (1998)

7. Kirchgraber, U., Palmer, K.J.: Geometry in the neighborhood of invariant manifolds of maps and flows and linearization. Pitman Research Notes 233. Essex: Longman 1990

8. Ortega, R., Tarallo, M.: Degenerate equations of pendulum-type. Commun. Contemp. Maths. 2, 127-149 (2000)

9. Palis, J., de Melo, W.: Geometric Theory of Dynamical Systems: An Introduction. Berlin: Springer 1982

10. Rabinowitz, P.H.: Heteroclinics for a reversible hamiltonian system. Ergod. Th. Dynam. Sys. 14, 817-829 (1994) 